\title{
Closing the Osteoporosis Care Gap in Hip Fracture Patients: An Opportunity to Decrease Recurrent Fractures and Hospital Admissions
}

\author{
Anna Byszewski, ${ }^{1,2}$ Genevieve Lemay, ${ }^{2}$ Frank Molnar, ${ }^{1,2}$ \\ Nahid Azad,, 2 and Seanna E. McMartin ${ }^{3}$ \\ ${ }^{1}$ The Geriatric Assessment Unit, The Ottawa Hospital, Civic Campus, 1053 Carling Avenue, Ottawa, ON, Canada K1Y 4 E9 \\ ${ }^{2}$ Faculty of Medicine, University of Ottawa, 451 Smyth Road, Ottawa, ON, Canada K1H 8M5 \\ ${ }^{3}$ School of Public Health, University of Alberta, 3-50 University Terrace, 112 Street Edmonton, AB, Canada T6G 2T4
}

Correspondence should be addressed to Anna Byszewski, abyszewski@ottawahospital.on.ca

Received 17 May 2011; Revised 3 July 2011; Accepted 4 July 2011

Academic Editor: E. M. Lewiecki

Copyright ( 2011 Anna Byszewski et al. This is an open access article distributed under the Creative Commons Attribution License, which permits unrestricted use, distribution, and reproduction in any medium, provided the original work is properly cited.

\begin{abstract}
Background. Falls and hip fractures are an increasing health threat to older people who often never return to independent living. This study examines the management of bone health in an acute care setting following a hip fracture in patients over age 65. Methods. Retrospective chart review of all patients admitted to a tertiary health facility who suffered a recent hip fracture. Results. 420 charts of patients admitted over the course of a year (May 1, 2007-April 31, 2008) were reviewed. Thirty-seven percent of patients were supplemented with calcium on discharge, and $36 \%$ were supplemented with vitamin D on discharge. Thirty-one percent were discharged on a bisphosphonate. Conclusion. A significant care gap still exists in how osteoporosis is addressed despite guidelines on optimal management. A call to action is required by use of multifaceted approaches to bridge the gap, ensuring that fracture risk is minimized for the aging population.
\end{abstract}

\section{Introduction}

Osteoporosis is a major threat to the well being of our aging population, both in Canada and worldwide. In Canada, approximately 1 in 4 women and one in 8 men suffer from this condition [1]. A woman reaching the age of 50 has a $40 \%$ life time risk of a fracture (at the wrist, vertebra or hip) [2]. Up to $95 \%$ of fractures in patents older than 75 who are hospitalized for a fracture, and $80-90 \%$ in those $60-74$ years of age, can be attributed to osteoporosis [3]. By 2050, the worldwide incidence of hip fractures is projected to increase by $310 \%$ in men and $240 \%$ in women [4].

Among osteoporotic fractures, those at the hip are associated with the highest morbidity and mortality, with about $20 \%$ dying and $50 \%$ becoming institutionalized within the first year of the fracture [5]. Those who have a hip fracture are at a greater risk of a second osteoporosis-related fracture [6]. Of all recurrent fractures occurring within 2 years after a fracture, $60 \%$ occurred during the first year and $40 \%$ during the second year. The absolute risk to develop an incident clinical fracture within 2 years after any clinical fracture is $10.8 \%$ [7].

The primary goal of therapy after a fragility fracture (a fracture resulting from mechanical forces that would not ordinarily cause fracture, such as a fall from standing height) should be to prevent future fractures, which is accomplished by slowing or stopping bone loss, maintaining bone strength, and minimizing or eliminating factors that contribute to fractures (e.g., falls).

Inadequate mean baseline calcium intake was found in $85 \%$ of subjects in six major osteoporosis trials [8]. As well, more than half of a sample of 1536 women with postmenopausal osteoporosis had serum 25(OH) D levels below those associated with secondary hyperparathyroidism [9]. A Finnish study found that post hip-fracture death can 
be decreased by $36 \%$ in females and $43 \%$ in males at 3 years by treatment with calcium and vitamin $\mathrm{D}$ after fracture. However, in women taking the regimen of a bisphosphonate with calcium and vitamin $\mathrm{D}$, the mortality at 3 years was decreased by $43 \%$ [10]. What is alarming is the fact that even less persistence with treatment is seen over time. Rabeda et al. reported that for 23,146 patients in Belgium, who had sustained hip fracture, only $6 \%$ were discharged on a bisphosphonate [4]. At 6 months, 2.6\% were taking their bisphosphonate and at 1 year, 3.6\%.

At this time, bisphosphonates are the accepted first-line therapy in fracture prevention for both men and postmenopausal women, together with supplementation of vitamin $\mathrm{D}$ and calcium, although new treatments are on the horizon [4]. The Canadian Clinical Practice Guidelines (CPGs) were initially published in 2002 and updated in 2006 and more recently in 2010, http://www.cmaj.ca/cgi/rapidpdf/ cmaj. 100771v1?ijkey=460251aca 1 ba674e2b5b01f8bbe0f1b0 80b75d8\&keytype2=tf_ipsecsha [11]. These guidelines clearly outline risk factor assessment, investigations, and management of fragility fractures $[12,13]$. In the most recent update, they clearly state that treatment of osteoporosis is justified regardless of the BMD level in patients who have already sustained a fragility fracture and are on prolonged glucocorticoid treatment (more than 3 months at $7.5 \mathrm{mg}$ or more of prednisone equivalent).

A systematic review concluded that the median rate of osteoporosis investigation in patients who had sustained a fragility fracture was $11 \%(0.5-32 \%)$, and fewer than $10 \%$ of patients were treated with antiresorptive therapy [14]. In a review of 298 patients with distal radius fracture, only $21.3 \%$ had a DXA scan ordered to investigate for osteoporosis [15].

The literature is emerging on the barriers to care. Several patient-related barriers of initiation of effective osteoporosis management have been identified: age, dementia, medical co-morbidities, polypharmacy, lack of adherence to treatment, post-operative delirium, language barriers, inadequate support, lack of access to care by a primary care physician, and social economic status [16].

Physician and system-related barriers have been acknowledged including lack of time, cost of resources for diagnosis, lack of knowledge and concern about osteoporosis treatment, and lack of clarity whose responsibility is it to initiate and promote osteoporosis care. Furthermore, inadequacy of communication between orthopedics and the family physician, insufficient means of transportation for patient followups, and uncertainties in applying diseasespecific guidelines to older patients with comorbidities contribute to these barriers [17]. One study reports that most orthopedic surgeons believe that while they are primarily responsible for the surgical care of their hip fracture patients, the management of osteoporosis is considered the responsibility of the primary care physician [18].

In a previous study performed in 1999-2000 at The Ottawa Hospital, Civic Campus, a tertiary care centre, we demonstrated a similar care gap as in published literature, in the management of osteoporosis in older hip fracture patients [19]. In this study of 147 patients over 65 years
TABle 1: Demographics $(n=420)$.

\begin{tabular}{lcc}
\hline \multicolumn{3}{c}{ Patient characteristics } \\
\hline Female & $N(\%)$ & $310(74 \%)$ \\
\hline \multirow{2}{*}{ Age } & Mean (Std) & $83.1(7.5)$ \\
& Median & 83 \\
& Range & $66-103$ \\
\multirow{2}{*}{ Average length of stay (days) } & Mean (Std) & $21.1(33.3)$ \\
& Median & 11 \\
& Range & $0-431$ \\
\hline
\end{tabular}

of age, admitted with a hip fracture, management included only $14 \%$ being supplemented with vitamin D, 15.6\% were supplemented with calcium, and $4.8 \%$ of the total sample were discharged on a bisphosphonate (Figure 3 ).

\section{Objective}

We report the results of this current follow-up study to evaluate how we are doing 5 years after the initial 2002 CPG were made available to the Canadian health care system, as compared to the initial study in 1999, and on the heels of the recent updated $2010 \mathrm{CPG}$. Strategies on how to bridge the gap in the most vulnerable population, those who suffered a hip fracture, in light of the recent 2010 osteoporosis guidelines, will be presented.

\section{Methods}

Retrospective chart review was conducted of all the hip fracture patients at The Ottawa Hospital (now combined Civic and General campuses) using a standardized questionnaire. Research ethics approval was obtained from The Ottawa Research Ethics Board.

\section{Results}

4.1. Demographics. The charts of 420 patients over the age of 65 years, admitted over the course of one year (May 1, 2007-April 30, 2008), were reviewed (Table 1). Of the patients, $74 \%$ were female and the mean age was 83 years. The average length of stay was 21 days with $82 \%$ being discharged from an orthopedic ward, further $6 \%$ from the Geriatric Assessment Unit (GAU) (Figure 1). Data on comorbidities was available only on 147 patients, with the most frequent being hypertension (58\%), previous history of falls (57\%), previous diagnosis of osteoporosis $(28 \%)$, cardiovascular disease $(27 \%)$, osteoarthritis $(23 \%)$, previous fracture $(22 \%)$, and cognitive impairment (28\%).

4.2. Patient Living Arrangements on Admission and Discharge. Of the 420 charts reviewed, the living arrangements upon admission were: $72.5 \%$ patients being admitted from home, $23.6 \%$ from nursing home, and 3.9\% from other settings. However, the discharge locations were quite different with only $15.8 \%$ patients discharged going home, $32.7 \%$ to a nursing home, $31.2 \%$ to a rehabilitation facility, and $20.3 \%$ 


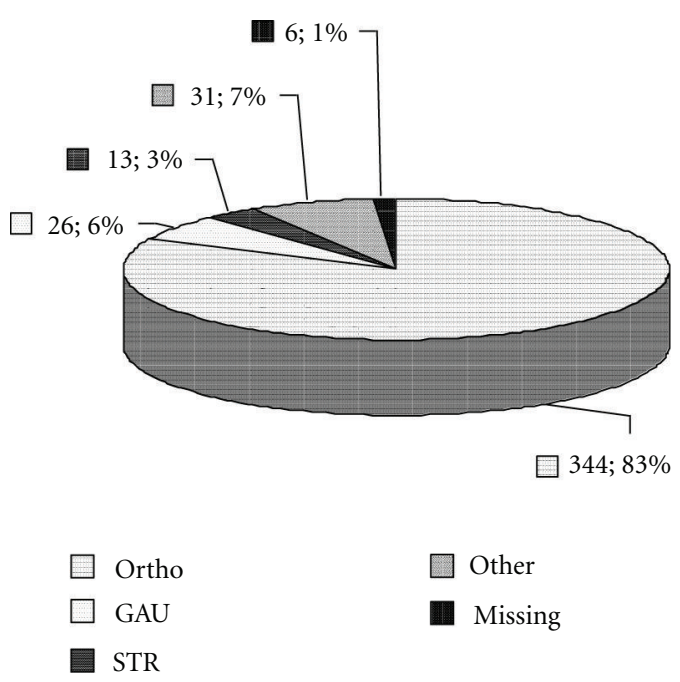

Figure 1: Ward location on discharge.

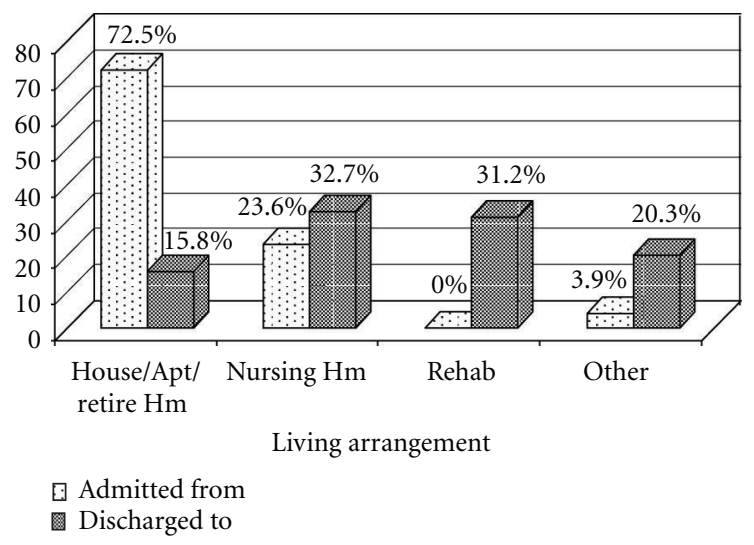

FIgURe 2: Patient living arrangement at admission and discharge.

to other settings (e.g., boarding home, other hospital) (Figure 2).

4.3. Use of Calcium Supplementation. On discharge (Figure 3), $37 \%$ of patients were started on calcium supplementation, with $17 \%$ already being on these supplements prior to admission, for a total of new supplementation of calcium of $20 \%$. Sixty-one percent were not supplemented with calcium on discharge. In $2 \%$ the discharge treatment did not include calcium, even though they were on it prior to admission.

4.4. Use of Vitamin D Supplementation. On discharge (Figure 3), 36\% of patients were started on vitamin D supplementation, with $14 \%$ of these patients being on vitamin $\mathrm{D}$ at time of admission, for a total of $21 \%$ new supplementation of vitamin D. Sixty-three percent were not recommended to start vitamin $\mathrm{D}$, and $1 \%$ were not continued on vitamin $\mathrm{D}$, even though it was listed in their admission medications.

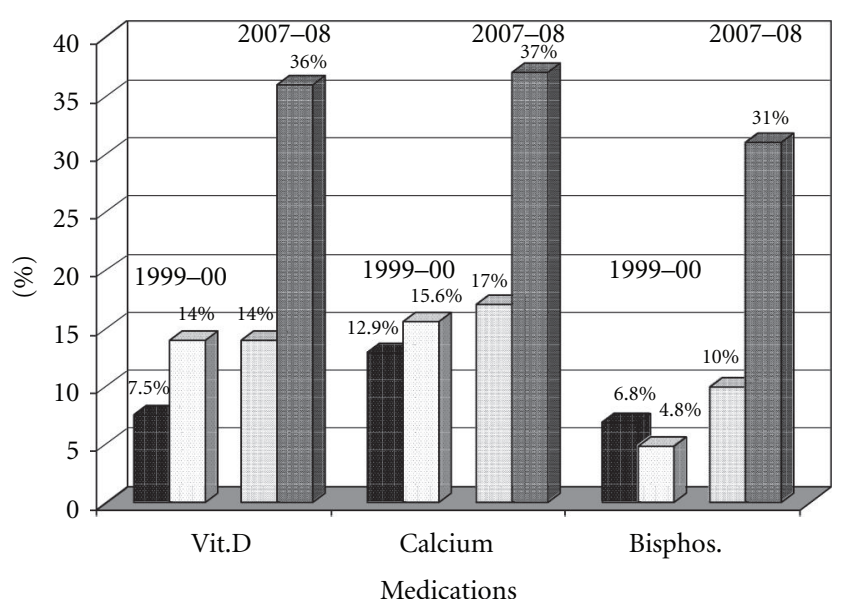

FIGURE 3: Medications at admission and discharge (as compared to 1999-2000 Study).

4.5. Use of Bisphosphonates. On discharge (Figure 3), 31\% of patients were started on a bisphosphonate (either alendronate $17 \%$, risedronate $10 \%$, or etidronate $4 \%$ ), with $10 \%$ already being on a bisphosphonate prior to admission, giving a $21 \%$ rate of new prescribing. Sixty eight percent were not on a bisphosphonate on discharge. In $1 \%$ bisphosphonate was discontinued on discharge.

4.6. Management on GAU Unit. A further subgroup analysis of the smaller sample $(n=26)$ of patients discharged from the GAU was performed (Figure 4), demonstrating that $100 \%$ of patients were discharged on calcium $(19 \%$ were on calcium preadmission) and vitamin D (12\% were on vitamin $\mathrm{D}$ on admission), and $77 \%$ were prescribed a bisphosphonate. Of these, $15 \%$ were on a bisphosphonate before admission, for an increase in prescribing of $62 \%$ of bisphosphonate treatment.

4.7. DXA Scan. On discharge, $7 \%$ of all patients had a DXA scan ordered. Of the GAU subgroup analysis $(n=26), 58 \%$ were prescribed a DXA scan.

\section{Discussion}

Despite several years since the publication of the original Canadian practice guidelines on osteoporosis management, in addition to other international guidelines, we are still struggling with managing this challenging health care issue. Osteoporosis management may not be viewed as a high priority during in-hospital care, leaving this to "outpatient investigation and management by primary care physicians," or to a "consultant service." There seems to be a general lack of awareness and ownership of the problem [20]. This is evident from our study as well, as treatment rates were generally greater on the GAU although a major limitation is the comparably smaller sample (344 versus 26). Following the initial study [19], several interventions were instituted on the GAU, such as staff education, osteoporosis discharge check list, and providing a patient education binder. This 
resulted in raising general awareness and a culture of managing osteoporosis and fracture risk.

A limitation of our study is that it is a retrospective chart review, so we were unable to explore the reasons why treatment was not initiated and therefore can only speculate. While $100 \%$ of patients on the GAU were discharged on calcium and vitamin D, only $77 \%$ were discharged on a bisphosphonate with $62 \%$ being new prescriptions. For those who were not treated, some of the barriers described above could have also played a role in lower adherence to guidelines.

Our study was also limited by lack of follow-up data to verify if treatment was commenced eventually by family physicians for those who left hospital on no treatment, and if the persistence was maintained. Previous studies with family physicians demonstrate that they are often unclear about the rationale for therapy and do not feel that they have the adequate resources, information and time to deal with this issue. In a small trial in a community-based setting after hip fracture, less than one-third of patients randomized to the control group received adequate care (including bone density measurement, bisphosphonate, vitamin D, calcium, or exercise prescription) despite being admitted to a tertiary hospital and having follow up with their family physician [20]. This discrepancy in care delivery contributes to the continued care gap in fracture prevention, especially for those patients admitted to hospital, who are often older, frail individuals with many co morbidities, often suffering from prolonged immobility, and barriers to seeking care following discharge, as outlined above.

Our data also suggests that the rate of DXA scans ordered on admission was low in the overall sample, although higher on the GAU (58\%). One approach to improve the adherence with therapy could involve ensuring that the result of the DXA report is sent to the family physician for follow-up.

Patients admitted to hospital with fragility fractures could be routinely supplemented with calcium and vitamin $\mathrm{D}$, given the prevalence of inadequate levels of calcium [8] and vitamin $\mathrm{D}$ [9] reported in the literature. The current total daily recommendations are now for $1200 \mathrm{mg}$ of elemental calcium and 800-2000 IU of vitamin D [11]. Educating patients as well is pivotal in managing osteoporosis and pamphlets on the subject matter with instructions, given on discharge, may help to bridge the current care gap. Gardner et al. [21] showed an improvement in osteoporosis treatment with their intervention group that went through discussion during hospitalization about osteoporosis, diagnosis, treatment, and series of questions to discuss further with their family physician. At 6 weeks after discharge from hospital, this intervention group was found to have osteoporosis addressed by family physician in $42 \%$ versus $19 \%$ in the regular treatment group. In another study, care pathways have been explored in a London, Ontario study for patients with hip fracture. The result showed improvement $(13.5 \%$ versus $72 \%$ ) with calcium and vitamin D treatment [22].

The role of Osteoporosis Nurse Specialists has been recognized to play an important role in identifying patients at risk [17]. They can assist in liaison of the multidisciplinary teams with staff emergency physicians, orthopedic surgeons,

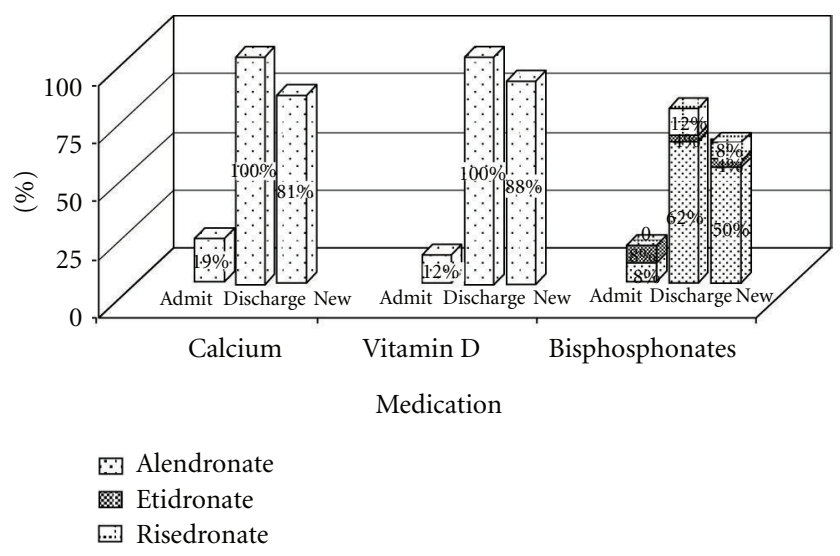

FIgURE 4: Medication at admission, discharge, and new prescriptions in GAU Group.

rehabilitation specialists, and primary care physicians. For example, Bogoch et al. [23] have championed a program for osteoporosis education, investigation and treatment in 349 patients (both inpatients and outpatients with fragility fracture in Toronto, Ontario, Canada). They were able to achieve a $96 \%$ rate of appropriate attention for osteoporosis in their Osteoporosis Exemplary Care program. This is an example of a coordinated program that has been rolled out to some regions of the province, but it requires resources and attention of local health authorities. Fracture clinics are beginning to utilize these resources, but they are often reserved for ambulatory outpatients.

In Ottawa, the Ontario Osteoporosis Screening program http://www.osteostrategy.on.ca/ has been active since 2007 and is run out of the fracture clinics. Unfortunately, hip fracture patients who are often older may not be accessing these services for the reason and barriers outlined above.

Although bisphosphonates are recognized as first-line therapy, concerns have been raised about potential side effects such as osteonecrosis of the jaw and proximal femur fractures. The overall risk is extremely low, especially given the grim prognosis of a hip fracture. Black et al. demonstrated the risk of proximal femur fractures, (as bone turnover may be decreased with long-term bisphosphonate therapy), to be 2.3 per 10,000 patient years [24]. The National Osteoporosis Foundation (NOF), in its clinical update online of July 2008, stated, "Results suggest that for most women, taking a 5-year drug holiday" after being on alendronate for 5 years does not increase fracture risk and might be advantageous [24]. Osteonecrosis of the jaw, which mainly affects those with malignancies being treated with intravenous forms of bisphosphonates, is estimated to have an incidence of 0.7 per 100,000 patient years [25]. Notwithstanding these concerns, treatment must be individualized.

The time of hospital admission is a prime opportunity to bridge this care gap, and systemic solutions need to be instituted to ensure that osteoporosis is addressed following a fragility fracture. Haynes et al. have proposed a checklist, for 
TABLE 2: Top ten suggested recommendations for all hip fracture patients over age 65.

(1) Supplement to total (dietary and prescribed) elemental calcium $1200 \mathrm{mg}$ daily (as average daily dietary calcium intake is around $600 \mathrm{mg}$, supplement with extra $600 \mathrm{mg}$, unless high dietary intake history)

(2) Supplement with vitamin D 800-2000 I.U. daily

(3) Consider first-line treatment with bisphosphonate-alendronate, risedronate, or i.v. zoledronic acid

(4) No need for DXA scan to initiate treatment, consider baseline DXA scan to assess bone density to follow response to treatment

(5) Assess and minimize fall risk and review all contributing risk factors

(6) Hip protectors if compliance can be ascertained (supervision, long term care setting)

(7) Patient and family education on osteoporosis, risk factors, importance of treatment to assure patient understanding, and improved compliance

(8) Encourage exercise-Tai Chi for balance and weight bearing if possible

(9) Develop specialized follow-up fracture clinics and orthogeriatric collaboration services for older hip fracture adults

(10) Followup DXA scan at the same location to asses bone density 1-3 years after the initiation of treatment to ensure compliance and response

use in surgery as a safety net to improve team communication and consistency of care [26]. Perhaps this can be adapted in other areas of our health care, including for older patients who suffered hip fractures. Table 2 lists recommendations for older hip fracture patients based on the 2010 guidelines.

\section{Conclusion}

Future research must focus on treatment evaluation and continuous quality improvement to achieve most effective measures for the implementation of the guidelines' recommendations and for improved compliance.

We require a call to action for a multifaceted, multidimensional model of care, to help address this care gap in our aging fracture susceptible population, at every point of contact, as many older patients may not have a primary care physician to follow up. Hopefully the next decade will see a widespread excellence of improved care models, as knowledge is gained, and other treatment options emerge.

\section{Key Points}

(1) Osteoporosis care gap continues in older patients with hip fractures: osteoporosis is underrecognized and undertreated in this population.

(2) Management should be instituted at time of hospitalization, including starting calcium, vitamin $\mathrm{D}$, and possibly a bisphosphonate, with treatment individualized for each older person given their comorbidities.

(3) Multidimensional interventions need to be implemented in addressing this health care threat to our aging population; for example, Osteoporosis Nurse Specialist led screening, follow up clinics, fall prevention clinics, exercise, and nutritional evaluation.

\section{Authors' Contributions}

Anna Byszewski and Nahid Azad contributed to the design of the study. Seanna E. McMartin was involved in the acquisition of the data. Anna Byszewski, Nahid Azad, Frank Molnar, and Genevieve Lemay contributed to the data analysis, interpretation of the data, and initial manuscript development. Frank Molnar and Nahid Azad provided additional critical revisions of the manuscript for important intellectual content. All authors approved the final version of the manuscript submitted for publication.

\section{Conflict of Interests}

The authors declare that they have no conflict of interests.

\section{Funding}

The summer studentship program that supported Seanna McMartin was funded through an unrestricted educational fund by Merck Frosst Canada.

\section{Acknowledgments}

The authors are grateful to Ann Cranney for critique of study design and manuscript preparation. James Jaffrey and Elham Sabri from the Ottawa Methods Centre, OHRI (Ottawa Health Research Institute) who provided assistance with data analysis. The authors also thank Sharon MacLaren for assistance with the manuscript preparation. Debbie Ayotte was instrumental with providing up-to-date literature review support.

\section{References}

[1] D. A. Hanley, R. G. Josse, and M. Bowyer, "Prevention and management of osteoporosis: consensus statements from the Scientific Advisory Board of the Osteoporosis Society of Canada. 1. Introduction," CMAJ, vol. 155, no. 7, pp. 921-923, 1996.

[2] J. A. Kanis, O. Johnell, A. Oden et al., "The risk and burden of vertebral fractures in Sweden," Osteoporosis International, vol. 15, no. 1, pp. 20-26, 2004.

[3] R. Goeree, B. O'Brien, L. Pettitt D' Cuddy, M. Ferraz, and J. Adachi, "An assessment of the burden of illness due to osteoporosis in Canada," Journal of Obstetrics and Gynaecology Canada, vol. 18, supplement, pp. 15-24, 1996. 
[4] V. Rabeda, J. Vanoverloop, V. Fabri et al., "Low incidence of osteoporosis treatment after hip fracture," Journal of Bone and Joint Surgery (American Volume), vol. 90, no. 10, pp. 21422148, 2008.

[5] S. R. Cummings and L. J. Melton, "Osteoporosis I: epidemiology and outcomes of osteoporotic fractures," The Lancet, vol. 359, no. 9319, pp. 1761-1767, 2002.

[6] C. M. Klotzbuecher, P. D. Ross, P. B. Landsman, T. A. Abbott III, and M. Berger, "Patients with prior fractures have an increased risk of future fractures: a summary of the literature and statistical synthesis," Journal of Bone and Mineral Research, vol. 15, no. 4, pp. 721-739, 2000.

[7] S. Van Helden, J. Cals, F. Kessels, P. Brink, G. J. Dinant, and P. Geusens, "Risk of new clinical fractures within 2 years following a fracture," Osteoporosis International, vol. 17, no. 3, pp. 348-354, 2006.

[8] R. P. Heaney, S. Magowan, X. Zhou, and S. Boonen, "Prevalence of low calcium intake in postmenopausal osteoporotic women: a need for supplementation (presentation M276)," in Proceedings of the 27th Meeting of American Society for Bone Mineral Research, Nashville, Tenn, USA, September 2005.

[9] M. F. Holick, E. S. Siris, N. Binkley et al., "Prevalence of vitamin D inadequacy among postmenopausal North American women receiving osteoporosis therapy," Journal of Clinical Endocrinology and Metabolism, vol. 90, no. 6, pp. 3215-3224, 2005.

[10] I. Nurmi-Lüthje, P. Lüthje, J. P. Kaukonen et al., "Postfracture prescribed calcium and vitamin D supplements alone or, in females, with concomitant anti-osteoporotic drugs is associated with lower mortality in elderly hip fracture patients: a prospective analysis," Drugs and Aging, vol. 26, no. 5, pp. 409-421, 2009.

[11] A. Papaioannou, S. Morin, A. M. Cheung et al., "2010 clinical practice guidelines for the diagnosis and management of osteoporosis in Canada: summary," CMAJ, vol. 182, no. 17, pp. 1864-1873, 2010.

[12] J. P. Brown and R. G. Josse, "2002 Clinical practice guidelines for the diagnosis and management of osteoporosis in Canada," CMAJ, vol. 167, no. 10, pp. S1-S34, 2002.

[13] J. P. Brown and M. Fortier, "Canadian consenus conference on osteoporosis 2006 update," American Journal of Obstetrics \& Gynecology, vol. 28, pp. S95-S112, 2006.

[14] V. Elliot-Gibson, E. R. Bogoch, S. A. Jamal, and D. E. Beaton, "Practice patterns in the diagnosis and treatment of osteoporosis after a fragility fracture: a systematic review," Osteoporosis International, vol. 15, no. 10, pp. 767-778, 2004.

[15] T. D. Rozental, E. C. Makhni, C. S. Day, and M. L. Bouxsein, "Improving evaluation and treatment for osteoporosis following distal radial fractures: a prospective randomized intervention," Journal of Bone and Joint Surgery - Series A, vol. 90, no. 5, pp. 953-961, 2008.

[16] J. A. Switzer, S. Jaglal, and E. R. Bogoch, "Overcoming barriers to osteoporosis care in vulnerable elderly patients with hip fractures," Journal of Orthopaedic Trauma, vol. 23, no. 6, pp. 454-459, 2009.

[17] T. R. Fried, M. E. Tinetti, and L. Iannone, "Primary care clinicians' experiences with treatment decision making for older persons with multiple conditions," Archives of Internal Medicine, vol. 171, no. 1, pp. 75-80, 2011.

[18] H. M. Khandwala, N. Kolla, and V. K. Grover, "Evaluation and treatment of osteoporosis in patients with a fragility hip fracture," Endocrine Practice, vol. 11, no. 6, pp. 370-375, 2005.

[19] A. M. Byszewski, A. Cranney, M. Man-Son-Hing, N. Azad, and S. Amos, "Evaluation of in-hospital management of frac- ture risk in older patients: a chart review study of tertiary prevention," Archives of Gerontology and Geriatrics, vol. 42, no. 3, pp. 319-328, 2006.

[20] G. Ioannidis, A. Papaioannou, W. M. Hopman et al., "Relation between fractures and mortality: results from the Canadian Multicentre Osteoporosis Study," CMAJ, vol. 181, no. 5, pp. 265-271, 2009.

[21] M. J. Gardner, R. H. Brophy, D. Demetrakopoulos et al., "Interventions to improve osteoporosis treatment following hip fracture: a prospective, randomized trial," Journal of Bone and Joint Surgery —Series A, vol. 87, no. 1, pp. 3-7, 2005.

[22] R. G. Crilly, M. Speechley, T. J. Overend, R. Mackenzie, S. Simon, and S. Cremer, "Evaluation of a care pathway in the initiation of calcium and vitamin D treatment of patients after hip fracture," Canadian Journal on Aging, vol. 28, no. 1, pp. 21-26, 2009.

[23] E. R. Bogoch, V. Elliot-Gibson, D. E. Beaton, S. A. Jamal, R. G. Josse, and T. M. Murray, "Effective initiation of osteoporosis diagnosis and treatment for patients with a fragility fracture in an orthopaedic environment," Journal of Bone and Joint Surgery-Series A, vol. 88, no. 1, pp. 25-34, 2006.

[24] "Update on Bisphosphonates FDA-Approved for Prevention and Treatment of Osteoporosis," National Osteoporosis Foundation Web Site, 2010, http://www.isesd.cv.ic.ac.uk.

[25] AAOMS, "American Association of Oral and Maxillofacial Surgeons position paper on bisphosphonate-related osteonecrosis of the jaws," Journal of Oral and Maxillofacial Surgery, vol. 65, no. 3, pp. 369-376, 2007.

[26] A. B. Haynes, T. G. Weiser, W. R. Berry et al., "A surgical safety checklist to reduce morbidity and mortality in a global population," The New England Journal of Medicine, vol. 360, no. 5, pp. 491-499, 2009. 


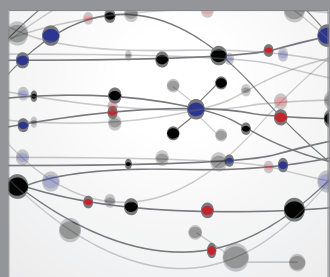

The Scientific World Journal
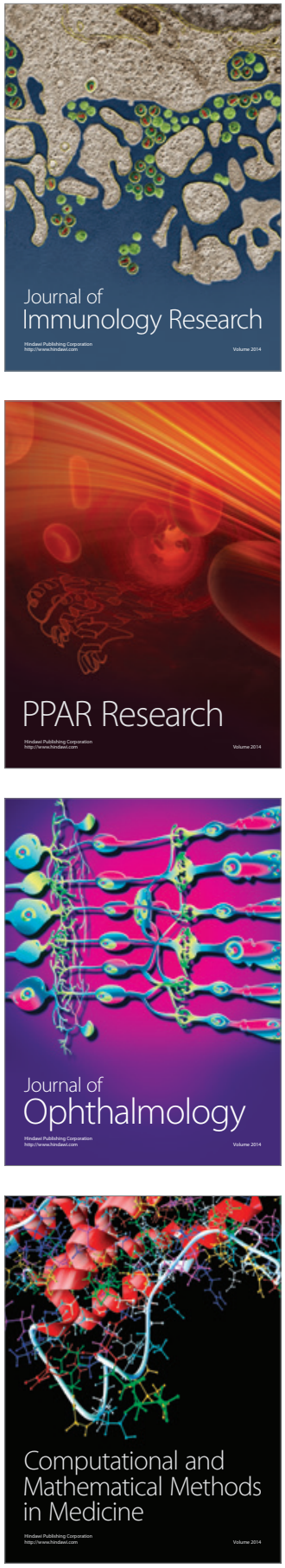

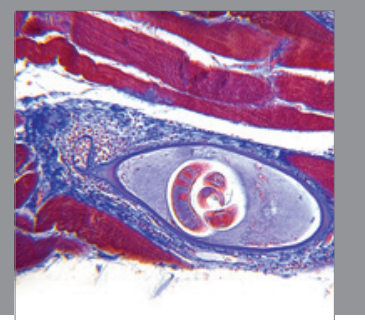

Gastroenterology

Research and Practice
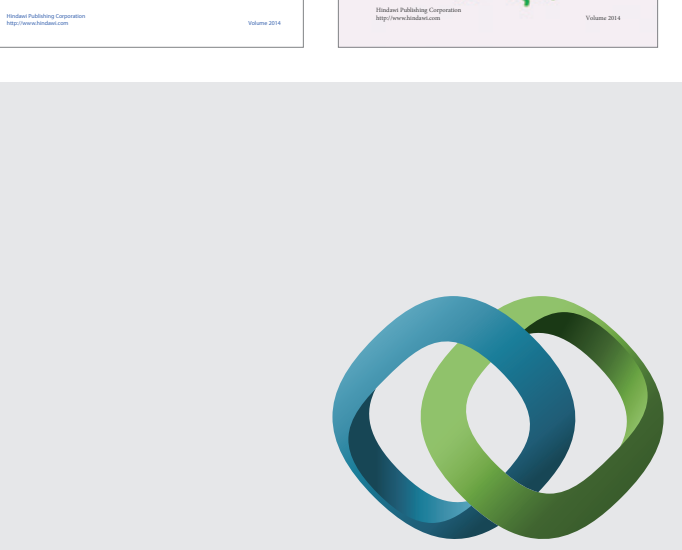

\section{Hindawi}

Submit your manuscripts at

http://www.hindawi.com
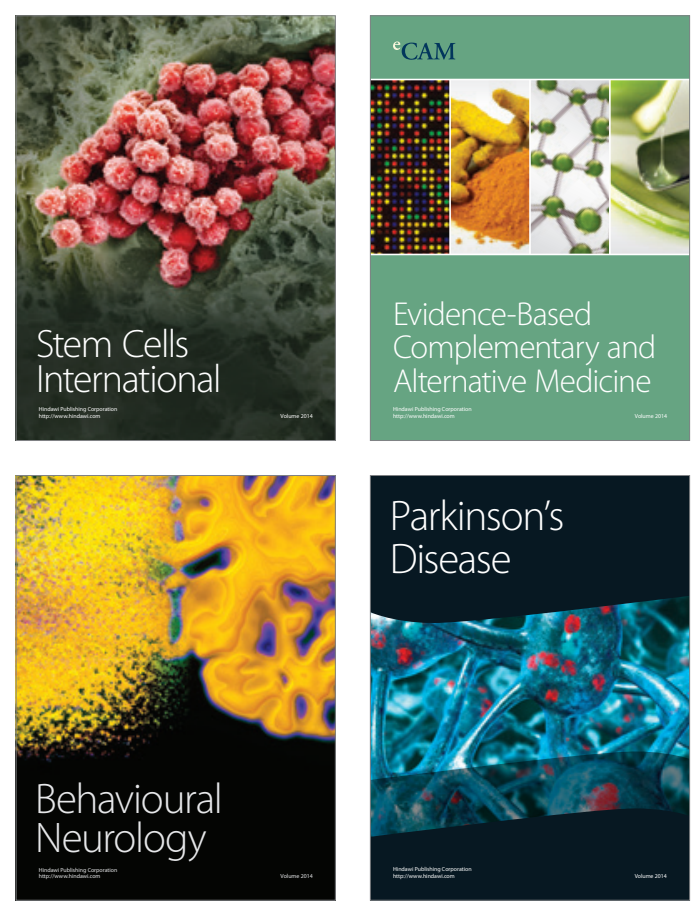

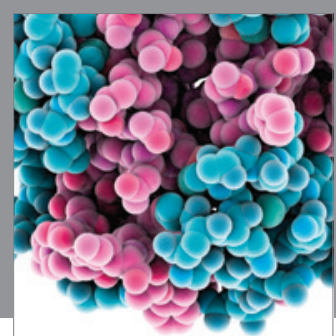

Journal of
Diabetes Research

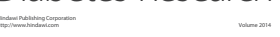

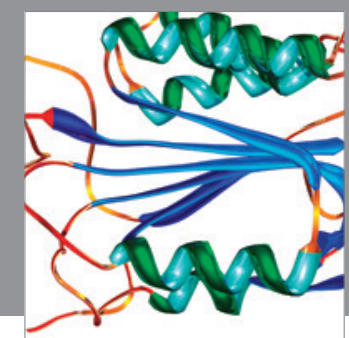

Disease Markers
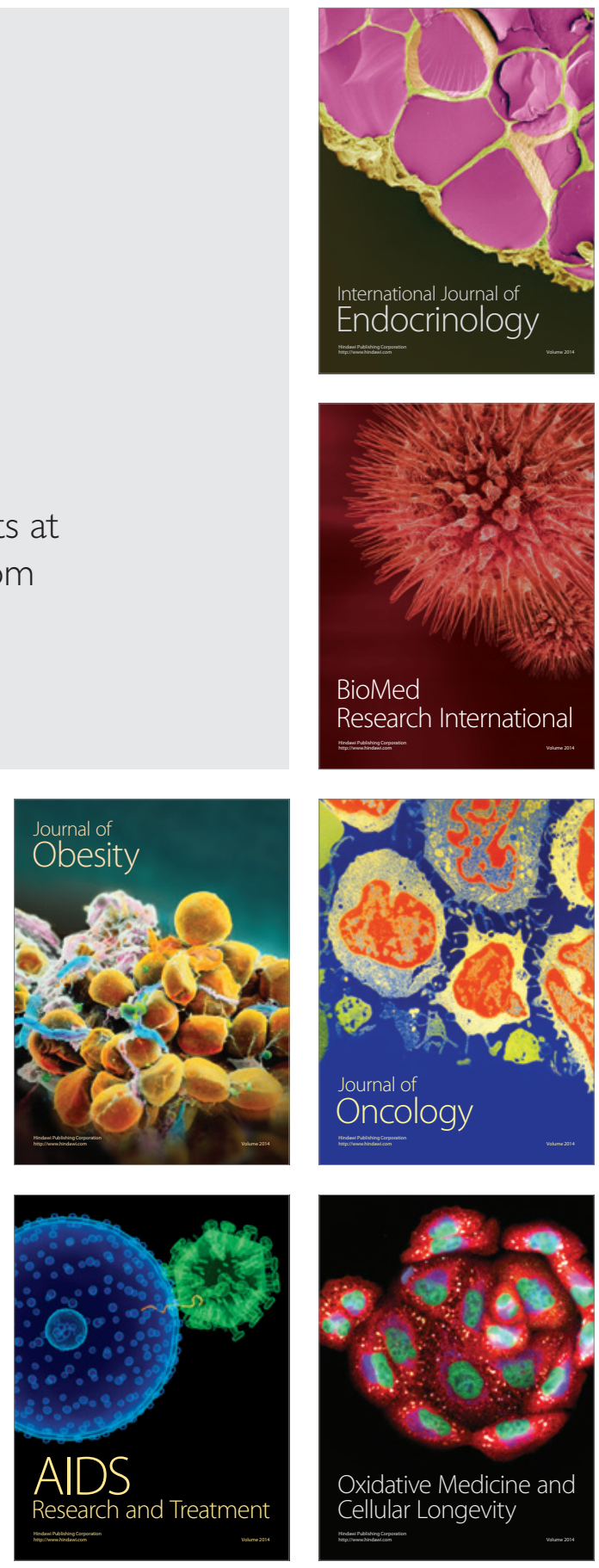